\title{
La cohabitation intergénérationnelle en entreprise : des stéréotypes prégnants à l'égard des seniors
}

Analyse de la situation belge

Nathalie Burnay

\section{OpenEdition}

Journals

Édition électronique

URL : http://journals.openedition.org/communicationorganisation/3543

DOI : 10.4000/communicationorganisation.3543

ISSN : 1775-3546

\section{Éditeur}

Presses universitaires de Bordeaux

\section{Édition imprimée}

Date de publication : 1 décembre 2011

Pagination : 71-86

ISBN : 978-2-86781-745-8

ISSN : $1168-5549$

Référence électronique

Nathalie Burnay, «La cohabitation intergénérationnelle en entreprise : des stéréotypes prégnants à l'égard des seniors », Communication et organisation [En ligne], 40 | 2011, mis en ligne le 01 décembre 2014, consulté le 19 avril 2019. URL : http://journals.openedition.org/

communicationorganisation/3543 ; DOI : 10.4000/communicationorganisation.3543 


\title{
La cohabitation intergénérationnelle en entreprise : des stéréotypes prégnants à l'égard des seniors Analyse de la situation belge
}

\author{
Nathalie Burnay'
}

\section{Vieillissement de la population versus faible taux d'emploi des seniors}

L'Union européenne vient de proclamer l'année 2012 comme étant l' "Année Européenne pour le Vieillissement Actif et la Solidarité entre les Générations $»^{2}$. À travers cette initiative, elle "encourage les décideurs politiques et toutes les parties prenantes à soutenir le vieillissement actif et à promouvoir une meilleure coopération et une plus grande solidarité entre les générations».

Cette déclaration s'inscrit dans une politique initiée depuis la fin des années 1990. Ainsi, en 1999, l'Union européenne érige la question des travailleurs vieillissants comme un élément majeur de l'agenda politique. Dans son rapport « Le marché du travail européen à la lumière des évolutions démographiques » de 1999, le groupe d'âge des 50-64 ans est identifié comme étant le réservoir principal de main-d'œuvre pour l'avenir. Le sommet de Lisbonne de mars 2000 propose aux États membres de conduire une politique active pour l'emploi afin d'atteindre un taux d'emploi de 70\% en 2010. Par le lancement de la Stratégie Europe 2020, qui remplace celle de Lisbonne, l'Union proclame à nouveau la nécessité d'une plus grande participation des travailleurs âgés sur le marché du travail et fixe le nouvel objectif de $75 \%$ de la population âgée de 20 à 64 ans en emploi.

Cependant, le bilan actuel est plus que mitigé ; seuls les pays nordiques et les Pays-Bas ont approché les objectifs fixés.

Pour transposer les recommandations européennes, la Belgique élabore, en 2005, le Pacte de solidarité entre les générations ${ }^{3}$. Ses objectifs sont multiples et

1. Nathalie Burnay est chargée de cours au Département de sciences politiques, sociales et de la communication des Facultés Universitaires Notre-Dame de la Paix (FUNDP) de Namur. Elle est également membre de l'Institut d'analyse du changement dans l'histoire et les sociétés contemporaines (IACCHOS) de I'Université catholique de Louvain (UCL) ; Nathalie.burnay@uclouvain.be ; Nathalie.burnay@fundp.ac.be.

2. Dossier « 2012 : Année Européenne pour le Vieillissement Actif et la Solidarité entre les Générations. Nous avons tous un rôle à jouer » (2011), Union Européenne, p 4-5.

3. Pacte de solidarité entre les générations - loi du 23 décembre 2005. 
visent notamment à favoriser une carrière plus longue et à freiner les départs prématurés du marché du travail... Malgré ces transformations des politiques publiques, la Belgique continue de se caractériser par un taux d'emploi des seniors extrêmement bas. Ainsi, en 2009, le taux d'emploi des 55-64 ans atteint seulement 35,3\% alors que la moyenne de l'Union Européenne est de $46 \%$ (Eurostat, 2010).

Les causes de ces faibles taux pour les seniors sont multifactorielles. I1 est dès lors difficile d'en cerner véritablement les contours, tant les effets d'interaction entrent en jeu. Ainsi, les différentes politiques d'emploi menées depuis la fin des années 1970 ont très certainement participé au développement des retraits anticipés du marché de l'emploi. En effet, durant les années 1970 et 1980, l'ensemble des pays européens subit de plein fouet la crise économique. Les taux de chômage s'envolent dans un contexte de restructuration drastique de l'industrie. Les politiques publiques, en Belgique notamment, montrent d'emblée une préférence marquée pour les jeunes chômeurs au détriment des travailleurs âgés ${ }^{4}$. Les travailleurs de 50 ans et plus sont encouragés par le gouvernement à se retirer du marché de l'emploi par le biais de préretraite, du chômage, de l'incapacité de travail et des pauses carrière. Certains aménagements liés à la pénibilité leur ont permis de suivre le même chemin. Ces politiques reposent sur un large consensus entre les instances politiques, les partenaires sociaux et les travailleurs : ces pratiques avaient pour objectif principal de laisser la priorité du marché de l'emploi aux jeunes par le dégraissage des aînés.

Par ailleurs, de nombreuses enquêtes ont mis en avant l'aspiration des travailleurs à quitter le monde professionnel bien avant l'âge légal de la retraite. Ainsi, deux récentes enquêtes belges confirment ces tendances. La première montre que l'âge idéal évoqué en Belgique pour souhaiter mettre un terme à son activité professionnelle se situe entre 59 et 60 ans $^{5}$; la seconde que $92 \%$ des travailleurs interrogés veulent mettre un terme à leur activité professionnelle avant 65 ans $^{6}$.

La dégradation des conditions de travail observée ces dernières années contribue également à vulnérabiliser les parcours professionnels, notamment par une augmentation du risque de problèmes de santé en fin de carrière ${ }^{7}$.

\footnotetext{
4. D. SIMOENS, " Hoe pijnloos is de crisis? Kritische analyse van de ontwikkeling van de wetgeving over de sociale zekerheidsprestaties (1976-1979) », Crisiswetgeving en social Zekerheid, Kluwer, Anvers, 1980, p. $70-82$.

5. M. ELCHARDUS, J. COHEN, Attitude et attentes en rapport avec la fin de carrière professionnelle, Rapport partiel $n^{\circ} 3$ : les déterminants de la fin de carrière, 2003.

6. N. BURNAY, "Volontary early Retirement: between desires and necessities », Pistes, 2008, vol.1, n², p. 1-19.

7. N. BURNAY, F. FALEZ, " Les impasses du maintien au travail. Production et gestion de trajectoires d'emploi sans issues : le cas de la sortie anticipée pour motif de santé ", Revue de l'Institut de Sociologie, 1-4, 2009, p. 59-73.
} 


\section{Stéréotypes sociaux à l'égard du travailleur âgé}

Parallèlement à ces nombreux facteurs, nombre d'auteurs ont insisté sur la présence de discriminations à l'égard du travailleur âgé sur le marché du travail. Apparu aux États-Unis en 1969, le terme âgisme est défini comme le processus par lesquel préjugés et discriminations se créent à l'encontre d'individus par le simple fait qu'ils sont plus âgés. Au niveau professionnel, deux types de discriminations fondées sur l'âge peuvent être identifiés : les discriminations directes ou indirectes ${ }^{8}$.

Les premières renvoient aux mesures qui visent les travailleurs âgés uniquement du fait de l'âge. On parlera de discrimination directe, par exemple, lorsque des limites d'âge figurent dans les offres d'emploi ou de formation, ou encore lorsqu'on impose l'âge de la retraite comme limite au-delà de laquelle il est interdit de travailler. Cette forme de discrimination a fait l'objet d'une réglementation particulière en Belgique (lois des 20 janvier et 25 février 2003) qui introduit des mécanismes de protection des travailleurs : il est interdit en cas de recrutement de personnel de faire référence à une limite maximale d'âge à partir de laquelle le candidat ne peut plus poser sa candidature ; il est également interdit dans le cadre de la sélection du personnel de fixer une limite d'âge maximale à partir de laquelle le candidat ne serait plus pris en considération pour une entrée en service.

Les discriminations indirectes sont des mesures qui, sans être directement liées à l'âge, affectent de manière disproportionnée et négative les travailleurs âgés par rapport à d'autres groupes d'âge ${ }^{9}$. Les stéréotypes ou préjugés présidant à la sélection de candidats à l'emploi font partie des formes de discrimination indirecte puisqu'ils sont susceptibles d'influencer les attitudes et les comportements tant de la personne qui les subit que de celle qui les exerce.

Ces formes de discriminations demeurent opérantes ${ }^{10}$, même lorsque les gouvernements tentent de légiférer. Ainsi, si les Etats-Unis ont été précurseurs en la matière, avec le vote de la loi réprimant la discrimination fondée sur l'âge dans le monde du travail (ADEA) dès 1967, les pratiques discriminatoires n'ont pas disparu. En témoignent, les milliers de dossiers rentrés auprès de la Commission de l'égalité et des chances dans l'emploi ou de recours devant les tribunaux américains ${ }^{11}$.

8. M. LELEU, Stéréotypes et travailleurs âgés. Le cas de la Belgique et des pays limitrophes : Luxembourg, Pays-Bas, Rapport pour le bureau international du travail, ILO, Genève, 2001 ; M.-C., AMAUGER-LATTES, « La discrimination fondée sur l'âge : une notion circonstancielle sous haute surveillance ", Retraite et société, 2007, vol.51, p. 28-41.

9. E. DRURY, Discrimination fondée sur l'âge exercée contre les travailleurs âgés de la communauté européenne. Une analyse comparative, Bruxelles, Eurolink Age, 1993.

10. J. MC GREGOR, «Stereotypes and older Workers: the New-Zealand Experience », Social Policy Journal of New Zealand, 2002, vol.18, p. 163-177 ; F. LACZKO, C. PHILLIPSON, Changing Work and Retirement. Social Policy and the Older Worker, Open University Press, Milton Keynes, 1991 ; C. ITZIN et C. PHILLIPSON, Age Barriers at Work, London, Metra, 1993

11. H.C. EGLIT, «L'âge dans le monde du travail aux Etats-Unis », Retraite et société, 2007, vol. 51, p. 44-75. 
L'hypothèse centrale de cet article repose sur la présence d'un lien étroit entre les formes de discrimination avérées et la prégnance de stéréotypes sociaux : les actes de discrimination étant fondamentalement liés à la présence d'une imagerie mentale défavorable aux seniors.

De nombreuses enquêtes sur les stéréotypes sociaux à l'égard du travailleur âgé ont été menées depuis les premières études américaines des années $1950^{12}$. Les stéréotypes étudiés dans les différentes enquêtes concernent à la fois l'évolution de la santé physique et mentale du travailleur âgé et son insertion professionnelle. Certains stéréotypes sont bien présents dans les esprits et prégnants : les difficultés physiques ${ }^{13}$, les difficultés cognitives ou mentales ${ }^{14}$, la résistance au changement ${ }^{15}$, les difficultés dans le rapport à la jeunesse ${ }^{16}$ ainsi que les difficultés d'apprentissage ${ }^{17}$ sont largement confirmées par les études.

\section{Méthodologie}

Cet article s'appuie sur des données récoltées auprès de 812 travailleurs ${ }^{18}$, répartis dans douze secteurs d'activité différents : industrie chimique, fabrication

12. W. KIRCHNER, T. LINDBOM, D.PATERSON, " Attitudes toward the Employment of Older People », Journal of Applied Psychology, 1952, vol. 36, n³, p. 154-156 ; W. KIRCHNER, M. DUNNETTE, "Attitudes toward Older Workers », Personnel Psychology, 1954, vol.7, p. 257-265 ; J. TUCKMAN, I. LORGE, « Attitudes toward older Workers ", Journal of Applied Psychology, 1952, vol. 36, n³, p. 149-153.

13. B. ROSEN, T.H. JERDEE, « Too Old or not too Old? », Harvard Business Review, 1977, p. 11-12 ; P. TAYLOR, A. WALKER, "Employers and Older Workers», Employment Gazette, 1993, vol. 101, n8, p. $371-378$; P. TAYLOR, A. WALKER, "The Ageing Workforce: Employers'Attitudes towards Older People», Work, Employment and Society, 1994, vol. 8, n4, p. 569-591 ; B. HASSELL, P. PERREWE, " An examination of Beliefs about Older Workers: do stereotypes still exist? », Journal of organizational Behaviour, 1995, vol. 16, n5, p. 457-468 ; S. PALONIEMI, T. TIKKANEN, "Victims of Ageist Attitudes-But how do the Older Workers themselves view their competences ", paper presented in ESREA research Network meeting Adult Education and the labour Market VI, Seville, October 2000 ; J. MC GREGOR, "Stereotypes and older Workers : the New-Zealand Experience ", Social Policy Journal of New Zealand, 2002, vol.18, p. 163-177.

14. B.S. AARONSON, "Personality stereotypes of Aging », Journal of Gerontology, 1996, vol. 21, p. 458-462 ; B. ROSEN, T.H. JERDEE, op. cit., p. $11-12$; P. TAYLOR, A. WALKER, « The Ageing Workforce : Employers' Attitudes towards Older People », op. cit.

15. B. ROSEN, T.H. JERDEE, op. cit., p. 11-12 ; P. TAYLOR, A. WALKER, "The Ageing Workforce : Employers' Attitudes towards Older People ", op. cit. ; P. LYON, D. POLLARD, "Perceptions of the Older Employee : is anything Changing? », Personnel Review, 1997, vol. 26, n²4, p. 245-252. ; C. REMERY, K. HENKENS, J. SCHIPPERS et P. EKAMPER, "Managing an aging Workforce and a tight labor market : views held by Dutch Employers ", Populations Research and Policy Review, 2003, vol. 22, p. 21-40.

16. P. TAYLOR, A.WALKER, "The Ageing Workforce : Employers' Attitudes towards Older People ", op. cit. ; B. HASSELL, P. PERREWE, "An examination of Beliefs about Older Workers : do stereotypes still exist? ", Journal of organizational Behaviour, 1995, vol. 16, n5, p. 457-468.

17. P. TAYLOR, A.WALKER, "The Ageing Workforce : Employers' Attitudes towards Older People ", op. cit. ; B. ROSEN, T.H. JERDEE, op. cit., p. $11-12$.

18. Recherche CAPA (2004-2008), financée conjointement par le Fonds social européen et le Service Public Fédéral Emploi, Travail et Concertation sociale, Division Humanisation du Travail. 
d'outils ou de machines, industrie automobile, construction, grande distribution, HORECA ${ }^{19}$, finances et assurances, recherche et développement, administration publique, enseignement, soins de santé et secteur social. L'objectif était de couvrir le plus largement possible le marché du travail. Les sujets ont été sélectionnés par des médecins du travail dans le cadre d'une consultation annuelle ${ }^{20}$.

La passation du questionnaire s'est déroulée en deux temps : dans un premier temps, le questionnaire était auto-administré par les répondants de manière à garantir un maximum d'autonomie dans les réponses recueillies ; dans un second temps, les médecins du travail ont vérifié le contenu des réponses en présence des répondants de manière à contrôler la fiabilité des données recueillies. Le questionnaire comportait deux parties, la première composée de questions permettant de cerner le travailleur répondant : variables sociodémographiques (âge, sexe, niveau d'étude, statut professionnel...), des variables de sociabilité (primaire, secondaire et tertiaire), des variables de qualité de vie et de niveau de satisfaction, des variables de trajectoire professionnelle ainsi que des variables de santé subjective et de stress. La seconde partie du questionnaire était composée de 33 items relatifs aux stéréotypes sociaux à l'égard du travailleur âgé. Cette liste a été construite en fonction de la littérature disponible sur le sujet ${ }^{21}$.

\section{Une première cartographie}

Une première analyse descriptive des données permet de nuancer la littérature existante. Ainsi, si les stéréotypes sociaux négatifs à l'égard du senior existent, notamment en termes d'incapacité à maittriser l'usage de nouvelles technologies et de résistance au changement, le tableau suivant montre combien l'imagerie mentale envers les seniors peut également s'exprimer positivement. Ainsi, les stéréotypes les plus partagés reposent sur une reconnaissance de leurs compétences : capacité à coacher les plus jeunes, compétences, fidélité à l'entreprise.

19. HORECA, principalement utilisé en Belgique, est le sigle du secteur d'activités de l'Hôtellerie, de la Restauration et des Cafés.

20. Les caractéristiques principales d'échantillon se trouvent reprises en annexe.

21. Annexe 2 : Présentation des stéréotypes sociaux liés à l'âge. 
Tableau 1. Fréquences des stéréotypes les plus prégnants et les moins prégnants $(n=816)$

\begin{tabular}{|l|c|c|c|}
\hline Nature du stéréotype & $\begin{array}{c}\text { D'accord } \\
(\%)\end{array}$ & $\begin{array}{c}\text { Pas d'accord } \\
(\%)\end{array}$ & $\begin{array}{c}\text { Pas d'avis } \\
(\%)\end{array}$ \\
\hline Les stéréotypes les plus prégnants & & & 13 \\
\hline Les travailleurs âgés doivent coacher les plus jeunes & 77 & 10 & 10 \\
Les travailleurs âgés sont les plus compétents & 77 & 13 & 16 \\
Les travailleurs âgés sont plus fidèles à l'entreprise & 72 & 12 & 11 \\
Les travailleurs âgés ont plus de difficultés à utiliser les nouvelles technologies & 68 & 21 & 11 \\
Les travailleurs âgés s'adaptent moins facilement au changement & 64 & 25 & 18 \\
Les travailleurs âgés prennent plus facilement des décisions seuls & 61 & 21 & \\
\hline Les stéréotypes les moins prégnants & & & \\
\hline Les travailleurs âgés sont plus souvent absents & & & 17 \\
Cela ne vaut plus la peine d'investir dans la formation des travailleurs âgés & 16 & 67 & 18 \\
Les travailleurs âgés sont plus souvent de mauvaise humeur & 14 & 68 & 15 \\
Les travailleurs âgés sont moins efficaces, moins productifs & 13 & 72 & 14 \\
Les travailleurs âgés coûtent trop cher par rapport à leur rendement & 8 & 78 & 18 \\
\hline
\end{tabular}

Ces premiers résultats, encourageants d'un point de vue managérial, doivent cependant être nuancés. Ainsi, le tableau suivant montre très clairement que les stéréotypes négatifs sont davantage présents dans la ligne hiérarchique que chez les ouvriers.

Tableau 2. Fréquence des stéréotypes négatifs en fonction du statut professionnel

\begin{tabular}{|c|c|c|}
\hline Nature du stéréotype & Ouvriers (\%) & Ligne hiérarchique (\%) \\
\hline Les travailleurs âgés ont besoin de plus de temps pour apprendre & 43.2 & 66.4 \\
\hline Les travailleurs âgés s'adaptent moins bien aux rythmes plus élevés & 51.1 & 63.3 \\
\hline Les travailleurs âgés ont plus de difficultés à utiliser les nouvelles technologies & 53.7 & 73.4 \\
\hline Les travailleurs âgés sont plus souvent absents & 12.3 & 16.5 \\
\hline Les travailleurs âgés s'investissent moins dans leur travail & 14.0 & 22.2 \\
\hline Cela ne vaut plus la peine d'investir dans la formation des travailleurs âgés & 10.9 & 15.2 \\
\hline Les travailleurs âgés sont plus lents & 16.9 & 26.9 \\
\hline
\end{tabular}

Ainsi, pour certains stéréotypes, un écart de plus de 20\% est observé entre les ouvriers et la ligne hiérarchique. Ces résultats confortent l'idée que des formes de discrimination à l'âge existent dans le monde professionnel et en particulier dans les fonctions les plus importantes.

\section{Une cartographie plus détaillée}

De manière à affiner ces premiers résultats, très intuitifs, il paraissait intéressant d'en comprendre les mécanismes et d'ainsi être capable d'en présenter une photographie plus complète. À cet effet, une analyse en composantes principales (Varimax Rotation, Kaiser's criterion) a été menée, en écartant, au préalable, les stéréotypes n'étant pas partagés par au moins $10 \%$ de la population. Seuls 28 stéréotypes ont ainsi été retenus dans l'analyse. 
Plus précisément, l'enjeu de cette analyse est d'effectuer un regroupement des stéréotypes et d'en comprendre la signification. C'est ainsi que sept facteurs, ou groupements, ont été identifiés; chacun possédant une logique propre et une cohérence interne, tant d'un point de vue statistique que du point de vue du sens attribué.

Facteur 1. Sens des responsabilités (13,75\% de la variance totale expliquée)

Ce premier facteur se caractérise par une opinion positive à l'égard des travailleurs âgés : ils sont plus méticuleux, plus compétents, plus loyaux envers leur entreprise, plus respectueux des règles et ont moins souvent d'accidents. Ce facteur confirme les analyses précédentes par son caractère positif.

Facteur 2. Adaptabilité (10\% de la variance totale expliquée)

Ce deuxième facteur renvoie à une incapacité à envisager les changements pour les travailleurs âgés. Il se caractérise par une lenteur dans la réalisation des tâches, une difficulté dans l'apprentissage de nouvelles compétences, une incapacité à gérer les nouvelles technologies et une mauvaise adaptation au changement.

Facteur 3. Aspect émotionnel (5,68\% de la variance totale expliquée)

Ce troisième facteur, moins significatif, se caractérise par des stéréotypes centrés sur des aspects plus émotionnels : les travailleurs âgés sont plus stressés, sont plus souvent déprimés et sont plus souvent absents.

Facteur 4. Les capacités cognitives (4,47\% de la variance totale expliquée)

Ce quatrième facteur regroupe les stéréotypes suivants : les travailleurs âgés ont besoin d'un temps de réaction plus important, ils éprouvent davantage de problème de concentration et de mémoire, ils sont plus souvent fatigués et leurs aptitudes physiques se détériorent.

Facteur 5. L'expérience professionnelle (4,42\% de la variance totale expliquée)

Ce cinquième facteur, positif, est centré sur l'expérience professionnelle des travailleurs âgés : ils prennent plus facilement des décisions seuls, ils peuvent moins suivre les directives, ils ont plus d'expérience et peuvent coacher les plus jeunes.

Facteur 6. La productivité (3,85\% de la variance expliquée)

Ce facteur est négatif et exprime deux sentiments à l'égard des travailleurs âgés : ils devraient laisser leur place aux plus jeunes et il n'est pas rentable d'investir dans leur formation.

Facteur 7. La fin de carrière (3,53\% de la variance expliquée)

Dans ce dernier facteur, les travailleurs âgés seraient plus heureux de partir en retraite avant 65 ans et également plus heureux de réduire leur temps de travail.

Le tableau suivant présente les contributions de chacun des stéréotypes aux facteurs. 


\begin{tabular}{|c|c|c|c|c|c|c|c|c|c|c|c|c|c|c|c|c|c|}
\hline 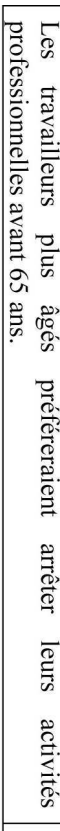 & 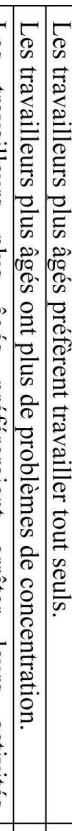 & 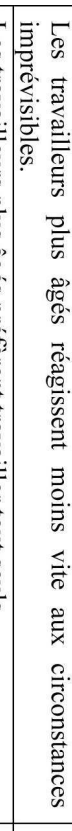 & 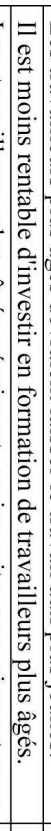 & 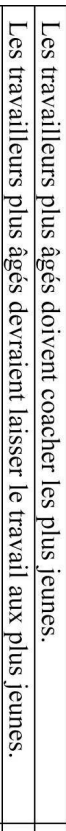 & 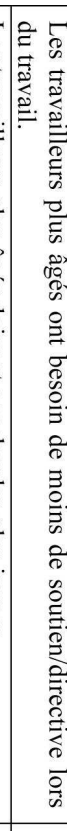 & 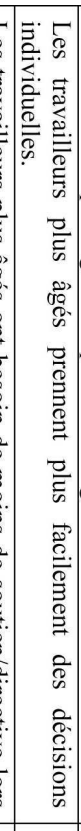 & 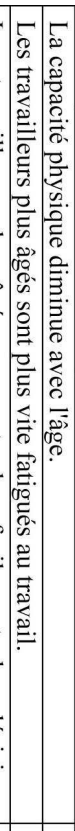 & 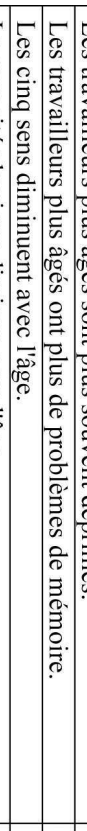 & 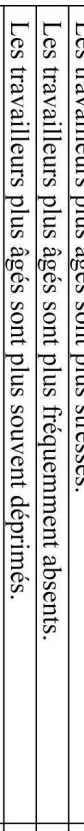 & 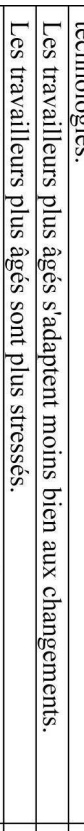 & 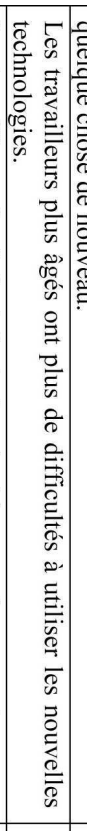 & 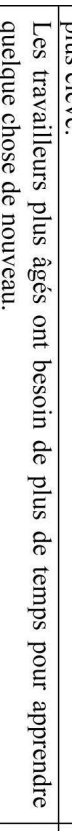 & 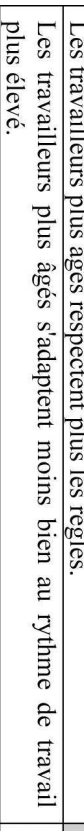 & 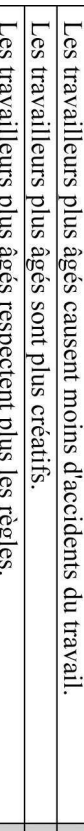 & 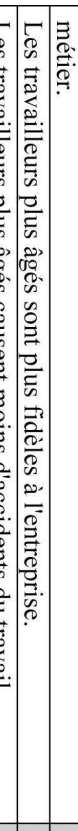 & 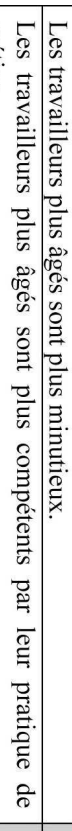 & \\
\hline$\therefore$ & $\begin{array}{ll}0 & 1 \\
0 & 0 \\
0 & 0 \\
0 & 0 \\
\infty & \\
0\end{array}$ & $\vec{v}$ & 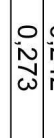 & $\begin{array}{ll}0 & 0 \\
\text { N } & 0 \\
\text { N } & 0 \\
\text { N } & 0 \\
0\end{array}$ & 官 & $\begin{array}{l}\dot{0} \\
\ddot{0} \\
0 \\
0\end{array}$ & $\begin{array}{lll}1 & 0 \\
\mathbf{N} & 0 \\
\mathbf{N} & 0 \\
& \mathbf{O} \\
\end{array}$ & 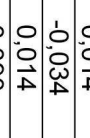 & $\begin{array}{lll}0 & 1 & \\
0 & 0 & 0 \\
0 & 0 & 0 \\
A & 1 & 1\end{array}$ & 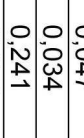 & $\begin{array}{l}\circ \\
\circ \\
\stackrel{0}{+}\end{array}$ & $\begin{array}{l}1 \\
0 \\
\text { No } \\
\text { - }\end{array}$ & $\begin{array}{l}1 \\
0 \\
0 \\
\vdots \\
1\end{array}$ & 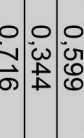 & 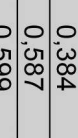 & ○̃ & u. \\
\hline $\begin{array}{l}0 \\
0 \\
0 \\
0 \\
\text { vil }\end{array}$ & 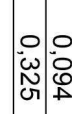 & & $\mid \begin{array}{l}0 \\
\sim \\
N \\
N \\
N\end{array}$ & 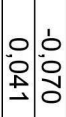 & $\overbrace{-\infty}^{0}$ & 足 & 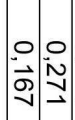 & $\begin{array}{ll}0 & 0 \\
\vec{N} & \stackrel{N}{N} \\
& 0\end{array}$ & 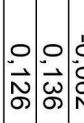 & 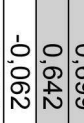 & & $\begin{array}{l}0 \\
\text { ज़ } \\
\text { 心 }\end{array}$ & $\begin{array}{l}0 \\
-1 \\
\text { ज } \\
\omega\end{array}$ & 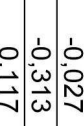 & 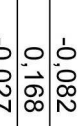 & $\begin{array}{l}\dot{0} \\
\dot{\vec{\omega}} \\
\infty\end{array}$ & \\
\hline $\begin{array}{l}0 \\
\vec{\infty} \\
\forall \\
\end{array}$ & $\begin{array}{ll}1 & 0 \\
0 & 0 \\
0 & 0 \\
0 & 0 \\
0\end{array}$ & : & $\begin{array}{l}\dot{0} \\
\vec{\omega} \\
N\end{array} \mid$ & 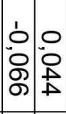 & $\begin{array}{l}0 \\
\frac{0}{\sigma} \\
\end{array}$ & $\begin{array}{l}0 \\
\stackrel{0}{+} \\
\stackrel{+}{+}\end{array}$ & \begin{tabular}{l|l}
0 & 0 \\
$\vec{\omega}$ & $\vec{N}$ \\
$\sigma$ & $\vec{D}$ \\
\end{tabular} & 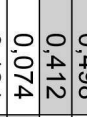 & 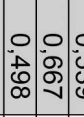 & 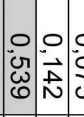 & & $\begin{array}{l}0 \\
8 \\
0 \\
0\end{array}$ & 怘 & 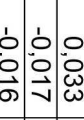 & $\begin{array}{lll}0 & 0 & 0 \\
\\
\omega\end{array}$ & $\stackrel{\circ}{\circ}$ & \\
\hline $\begin{array}{l}0 \\
0 \\
0 \\
0\end{array}$ & $\begin{array}{lll}0 & 1 \\
\circ & 0 \\
\circ & 0 \\
N & 0 \\
N & 0\end{array}$ & 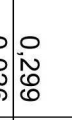 & $\mid \begin{array}{l}0 \\
0 \\
0 \\
\omega \\
\omega \\
\vdots\end{array}$ & $\begin{array}{ll}0 & 0 \\
: & \circ \\
0 & 0 \\
0 & 0 \\
\end{array}$ & 主 & 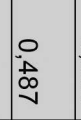 & 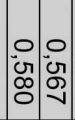 & \begin{tabular}{ccc}
0 & 0 \\
\hdashline & 0 \\
& $\infty$ \\
$\sigma$ & 0 \\
0 & 1
\end{tabular} & 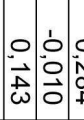 & 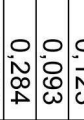 & 品 & $\begin{array}{l}\text { O } \\
\text { N } \\
\text { W }\end{array}$ & $\begin{array}{l}0 \\
\stackrel{N}{N}\end{array}$ & 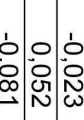 & 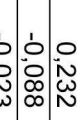 & 。 & \\
\hline o & $\mid \begin{array}{lll}0 & 0 \\
0 & 0 \\
0 & 0 \\
& 0\end{array}$ & a & $\begin{array}{l}0 \\
0 \\
0 \\
0 \\
0 \\
0\end{array}$ & 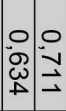 & 递 & 옹 & 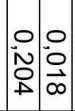 & $: 0$ & 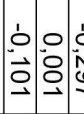 & 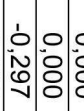 & 웅 & $\begin{array}{l}\dot{1} \\
0 \\
\infty\end{array}$ & $\begin{array}{l}0 \\
\stackrel{0}{\circ} \\
-1\end{array}$ & 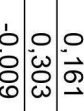 & 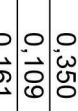 & $\begin{array}{l}\stackrel{0}{\vec{\Delta}} \\
\dot{\vec{\infty}}\end{array}$ & \\
\hline$\stackrel{O}{\vec{A}}$ & 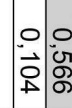 & 号 & $\mid \begin{array}{l}1 \\
0 \\
N \\
\vdots \\
\vdots\end{array}$ & $\begin{array}{ll}\circ & 0 \\
\circ & \circ \\
0 & 0 \\
0 & 0 \\
\infty & 0\end{array}$ & $\mid \begin{array}{l}0 \\
\omega \\
\vec{\omega} \\
0\end{array}$ & 웅 & 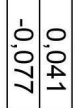 & 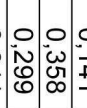 & $\begin{array}{lll}0 & 0 & \\
-\vec{A} & 0 & 0 \\
\vec{A} & 0 & 0 \\
\end{array}$ & $\begin{array}{lll}0 & 0 \\
0 & 0 & 0 \\
0 & 0 & 0\end{array}$ & $\begin{array}{l}\stackrel{0}{\overrightarrow{0}} \\
\overrightarrow{0}\end{array}$ & $\stackrel{\circ}{\stackrel{O}{\vec{N}}}$ & $\begin{array}{l}1 \\
0 \\
0 \\
0 \\
0\end{array}$ & 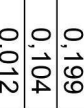 & 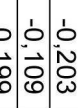 & $\begin{array}{l}0 \\
0 \\
0 \\
0\end{array}$ & \\
\hline$\therefore$ & 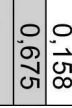 & in & $\mid \begin{array}{l}1 \\
0 \\
0 \\
0 \\
\infty\end{array}$ & $\begin{array}{lll}0 & 0 \\
0 & 0 \\
0 & 0 \\
N & \vec{\nabla} \\
N\end{array}$ & \begin{tabular}{l}
1 \\
0 \\
0 \\
0 \\
0 \\
\hdashline
\end{tabular} & 吕 & \begin{tabular}{|l|l|}
0 & 0 \\
$\overrightarrow{0}$ & 0 \\
0 & 0 \\
0 & 0 \\
\end{tabular} & 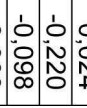 & 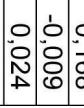 & 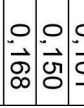 & $\stackrel{0}{\overrightarrow{0}}$ & $\begin{array}{l}0 \\
0 \\
\omega \\
\end{array}$ & : & 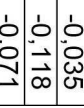 & 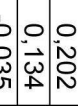 & $\stackrel{0}{\overrightarrow{\vec{v}}}$ & $\vec{\Delta}$ \\
\hline
\end{tabular}


L'analyse en composantes principales réalisée précédemment permet de pouvoir travailler à partir d'un regroupement de stéréotypes. Seuls les deux principaux facteurs seront pris en considération dans cette section (en rapport avec l'évolution du \% de variance expliquée). Il s'agira d'en comprendre les mécanismes et d'identifier des profils de travailleurs porteurs de ces représentations. Pour ce faire, des modèles de régression multiples ont été construits en prenant chaque facteur comme variable dépendante. Ces analyses permettent alors de mesurer l'impact de différentes variables sociodémographiques et professionnelles sur chaque facteur. Le tableau 4 présente les résultats de ces régressions.

Le premier facteur est composé de stéréotypes positifs où le travailleur âgé est perçu comme plus minutieux, plus compétent dans la pratique de son métier, plus créatif et plus respectueux des règles.

Lâge constitue une variable significative pour comprendre la construction de ces stéréotypes : plus l'on avance en âge, plus on partage ces stéréotypes. Cette relation peut s'expliquer par une certaine forme de proximité de l'expérience. À l'inverse, les cadres supérieurs, les travailleurs en bonne situation financière et en bonne santé sont plus critiques sur cette dimension positive. Le secteur tertiaire semble également moins propice au développement de cette imagerie positive à l'égard des seniors.

Le deuxième facteur comporte, quant à lui, une connotation négative. Il regroupe des stéréotypes tels que la difficulté d'adaptation, la lenteur dans l'apprentissage et l'incapacité à gérer les nouvelles technologies. À nouveau, l'âge a un réel impact : plus le travailleur est âgé, moins il partage ces images mentales. Ces stéréotypes sont plutôt l'apanage des cadres inférieurs et supérieurs, des travailleurs ayant un bagage scolaire plus important, en très bonne situation financière, en bonne santé et très stressés. De nouveau, le secteur des services se distingue par une position plus sévère à l'égard des seniors. 
Tableau 4. Régressions multiples sur les deux principaux facteurs

\begin{tabular}{|c|c|c|c|c|}
\hline \begin{tabular}{|l} 
\\
Groupes d'âge
\end{tabular} & \multicolumn{2}{|c|}{$\begin{array}{c}\begin{array}{c}\text { Sens des } \\
\text { responsabilités }\end{array} \\
(\mathrm{n}=772)\end{array}$} & \multicolumn{2}{|c|}{$\begin{array}{c}\begin{array}{c}\text { Moins grande } \\
\text { adaptabilité }\end{array} \\
\quad(n=772)\end{array}$} \\
\hline Moins de 34 ans & \multicolumn{2}{|c|}{-} & \multicolumn{2}{|c|}{ - } \\
\hline 35 à 44 ans & 0,156 & $* * *$ & $-0,032$ & (n.s.) \\
\hline 45 à 54 ans & 0,408 & $* * *$ & $-0,178$ & **** \\
\hline 55 ans et plus & 0,453 & $* * *$ & $-0,166$ & $* * *$ \\
\hline \multicolumn{3}{|l|}{ Genre } & \multirow{3}{*}{\multicolumn{2}{|c|}{ - }} \\
\hline Homme & \multicolumn{2}{|c|}{-} & & \\
\hline Femme & $-0,038$ & (n.s.) & & \\
\hline \multicolumn{5}{|l|}{ Statut professionnel } \\
\hline Ouvrier & \multicolumn{2}{|c|}{-} & \multicolumn{2}{|c|}{-} \\
\hline Employé & $-0,034$ & (n.s.) & 0,047 & (n.s.) \\
\hline Cadre inférieur & $-0,042$ & (n.s.) & 0,108 & $* *$ \\
\hline Cadre supérieur & $-0,124$ & ** & 0,120 & ** \\
\hline \multicolumn{5}{|l|}{\begin{tabular}{|l|} 
Niveau d'études \\
\end{tabular}} \\
\hline Primaire, secondaire inférieur & \multicolumn{2}{|c|}{-} & \multicolumn{2}{|c|}{-} \\
\hline Secondaire supérieur & $-0,080$ & (n.s.) & 0,136 & $* * * *$ \\
\hline Supérieur ou universitaire & $-0,068$ & (n.s.) & 0,222 & $* * *$ \\
\hline \multicolumn{5}{|l|}{ Situation financière } \\
\hline (Très) difficile & \multicolumn{2}{|c|}{-} & \multicolumn{2}{|c|}{-} \\
\hline Ni bonne ni mauvaise & $-0,080$ & $* *$ & 0,036 & (n.s.) \\
\hline (Très) bonne & $-0,118$ & * & 0,133 & ** \\
\hline \multicolumn{5}{|l|}{ Santé } \\
\hline Mauvaise ou convenable & \multicolumn{2}{|c|}{-} & \multicolumn{2}{|r|}{. } \\
\hline Bonne & 0,206 & $* * *$ & $-0,199$ & $* * *$ \\
\hline Très bonne ou excellente & 0,015 & (n.s.) & 0,014 & (n.s.) \\
\hline \multicolumn{5}{|l|}{ Stressé } \\
\hline Pas du tout & \multicolumn{2}{|c|}{-} & \multicolumn{2}{|c|}{-} \\
\hline Un peu & 0,007 & (n.s.) & 0,052 & (n.s.) \\
\hline Beaucoup & 0,060 & (n.s.) & 0,171 & **** \\
\hline \multicolumn{5}{|l|}{ Secteur professionnel } \\
\hline Secondaire (industries) & \multicolumn{2}{|c|}{-} & \multicolumn{2}{|c|}{-} \\
\hline Tertiaire (services) & $-0,121$ & $* * *$ & 0,124 & $* * *$ \\
\hline $\mathbf{R}$ & \multicolumn{2}{|c|}{0,449} & \multicolumn{2}{|c|}{0,416} \\
\hline $\mathbf{R}^{2}$ & \multicolumn{2}{|c|}{0,201} & & 173 \\
\hline Anova (signification du modèle) & & & & 000 \\
\hline
\end{tabular}




\section{Enguisedeconclusion:une dimension organisationnelleetcommunicationnelle}

Les analyses menées dans le cadre de cet article ont permis de cerner la présence de stéréotypes à l'égard des travailleurs âgés, confirmant par-là les résultats obtenus par les enquêtes déjà menées sur le sujet, tout en les nuançant. Ainsi, la présence de stéréotypes positifs apparaît comme un élément novateur dans le débat scientifique : les travailleurs âgés sont alors perçus comme pouvant constituer une ressource importante pour le monde du travail. Ce sont davantage les travailleurs, plus âgés, peu qualifiés, peu formés et issus du monde industriel qui en sont porteurs. Ils symbolisent toute la richesse de l'expérience, du bagage expérientiel des seniors.

Mais à l'inverse, et de manière toute aussi prégnante, les stéréotypes négatifs à l'encontre des travailleurs âgés hantent le monde du travail. Ils sont portés par une jeune génération, plus formée, plus qualifiée et un secteur des services plus virulent. La présence de ces stéréotypes témoigne d'une difficulté rencontrée par les seniors à s'adapter, à se plier aux exigences de modernisation. Ils se retrouvent dans l'ensemble des enquêtes menées sur ce sujet. Ainsi, quels que soient les contextes nationaux, ces stéréotypes demeurent prégnants et jouent à l'encontre des travailleurs plus âgés.

Ces résultats posent à la fois des questions organisationnelles et communicationnelles.

Ainsi, d'un point de vue organisationnel, les stéréotypes négatifs portés à l'encontre des seniors pèsent sur les destins professionnels et sur la construction des carrières : difficultés d'embauche des seniors, déficit de formation proposée, promotions bloquées... Lâge devient alors un handicap insurmontable pour le travailleur et une ressource encombrante pour l'organisation. Pour lutter contre cette forme de discrimination, un changement de perspective s'impose, une transformation des cadres de référence. Ainsi, l'entrée par l'âge est elle-même génératrice d'une forme de labelling. S'impose alors une réflexion sur la construction des carrières, des parcours de vie, des trajectoires professionnelles, transformant une logique transversale en une logique longitudinale. Ce n'est que par ce renversement de mentalité qu'une lutte efficace contre les discriminations pourra s'effectuer. Il s'agit donc de pouvoir déployer une véritable gestion prévisionnelle des ressources humaines, une gestion des âges, pensée dans un souci de développement des compétences et des ressources humaines.

D'un point de vue communicationnel, la présence de stéréotypes sociaux négatifs à l'encontre du senior nécessite un travail d'information et de prévention afin de démystifier les soi-disant carences du senior. Ainsi, la littérature montre qu'il existe peu de véritables déficits physiologiques avant l'âge de 70 ans ; que certes, l'apprentissage des seniors est plus lent, mais davantage opérant que chez les plus jeunes; qu'il existe des stratégies d'adaptation ou de compensation individuelles ou collectives pour s'adapter 
aux exigences du poste... Toutes ces nuances permettent de relativiser les soi-disant effets dévastateurs de l'âge $e^{22}$.

Il est par ailleurs évident que l'organisationnel et le communicationnel se renforceront mutuellement. Par exemple, la résistance au changement et le manque d'adaptation aux nouvelles technologies peuvent se résoudre plus que partiellement si le travailleur y est préparé tout au long de son parcours professionnel. Encore faut-il lui en donner l'opportunité et le soutenir dans la démarche... Ce n'est qu'à ce prix que l'on pourra rendre aux seniors une véritable place dans la structure et dans la société...

\section{BIBLIOGRAPHIE}

AARONSON B.S., « Personality stereotypes of Aging », Journal of Gerontology, 1996, vol.21, p. 458-462.

AMAUGER-LATTES M.-C., " La discrimination fondée sur l'âge : une notion circonstancielle sous haute surveillance ", Retraite et société, 2007, vol.51, p. 28-41.

BURNAY N., «Volontary early Retirement : between desires and necessities », Pistes, 2008, vol.1, no2, p. 1-19.

BURNAY N., FALEZ F., « Les impasses du maintien au travail. Production et gestion de trajectoires d'emploi sans issues : le cas de la sortie anticipée pour motif de santé ", Revue de l'Institut de Sociologie, 2009, vol. 1-4, p. 59-73.

CRESPO S., BEAUSOLEIL J., La sortie anticipée d'activité des travailleurs de 45 à 64 ans, Fiche techniques, Montréal, collection INRS Culture et Société, 1999.

DRURY E., Discrimination fondée sur l'âge exercée contre les travailleurs âgés de la communauté européenne. Une analyse comparative, Bruxelles, Eurolink Age, 1993.

EGLIT H.C., " Lâge dans le monde du travail aux Etats-Unis », Retraite et société, 2007, vol. 51, p. 44-75.

ELCHARDUS M., COHEN J., Attitude et attentes en rapport avec la fin de carrière professionnelle, Rapport partiel $\mathrm{n}^{\circ} 3$ : les déterminants de la fin de carrière, 2003.

GUILLEMARD A.M., L'âge de l'emploi : les sociétés à l'épreuve du vieillissement, Paris, Armand Colin, 2003.

HASSELL B., PERREWE P., «An examination of Beliefs about Older Workers : do stereotypes still exist? », Journal of organizational Behaviour, 1995, vol. 16, n 5, p. 457-468.

ITZIN C., PHILLIPSON C., Age Barriers at Work, London, Metra, 1993.

KILBOM A., «Evidence-based programs for the prevention of early exit from work », Experimental Aging Research, 1999, vol. 25, n4, p. 291-299.

KIRCHNER W., LINDBOM T., PATERSON D., "Attitudes toward the Employment of Older People ", Journal of Applied Psychology, 1952, vol. 36, n³, p. $154-156$.

22. S. VOLKOFF, F. MOLINIÉ et A. JOLIVET, Efficaces à tout âge ? Evolution démographique et activités de travail, Centre d'études de l'emploi, 2000, dossier 16. 
KIRCHNER W., DUNNETTE M., «Attitudes toward Older Workers », Personnel Psychology, 1954, vol. 7, p. 257-265.

LACZKO F., PHILLIPSON C., Changing Work and Retirement. Social Policy and the Older Worker, Open University Press, Milton Keynes, 1991.

LELEU M., Stéréotypes et travailleurs âgés. Le cas de la Belgique et des pays limitrophes: Luxembourg, Pays-Bas, Rapport pour le bureau international du travail, ILO, Genève, 2001.

LYON P., POLLARD D., "Perceptions of the Older Employee : is anything Changing? ", Personnel Review, 1997, vol. 26, n4, p. 245-252.

MC GREGOR J., "Stereotypes and older Workers : the New-Zealand Experience », Social Policy Journal of New Zealand, 2002, vol. 18, p. 163-177.

OCDE, Vieillissement et politiques de l'emploi : Belgique, Paris, Author, 2003.

PALONIEMI S., TIKKANEN T, «Victims of Ageist Attitudes- But how do the Older Workers themselves view their competences ", paper presented in ESREA research Network meeting Adult Education and the labour Market VI, Seville, October 2000.

REMERY C., HENKENS K., SCHIPPERS J. et EKAMPER P., « Managing an aging Workforce and a tight labor market : views held by Dutch Employers ", Populations Research and Policy Review, 2003, vol. 22, p. 21-40.

ROSEN B., JERDEE T.H., «The Nature of Job-related age Stereotypes ", Journal of Applied Psychology, 1976, vol. 61, n 4, p. 428-432.

ROSEN B., JERDEE T.H., "Too Old or not too Old? », Harvard Business Review, 1977, p. 11-12.

SIMOENS D., "Hoe pijnloos is de crisis? Kritische analyse van de ontwikkeling van de wetgeving over de sociale zekerheidsprestaties (1976-1979) », Crisiswetgeving en social Zekerheid, Kluwer, Anvers, 1980, p. 70-82.

TAYLOR P., WALKER A., « Employers and Older Workers », Employment Gazette, 1993, vol. 101, n8, p. 371-378.

TAYLOR P., WALKER A., «The Ageing Workforce : Employers' Attitudes towards Older People », Work, Employment and Society, 1994, vol. 8, n 4, p. 569-591.

TAYLOR P., WALKER A., "Employers and Older Workers : attitudes and Employment practices ", Ageing and Society, 1998, vol. 18, p. 641-658.

TUCKMAN J., LORGE I., "Attitudes toward older Workers ", Journal of Applied Psychology, 1952, vol. 36, n³, p. 149-153.

VAN DER VELDEN J., "Age-conscious social policy », dans SNEL J., CREMER R. (dir.), Work and Aging: a European prospective, London, Taylor and Francis, 1993, p. 353-364.

VOLKOFF S., MOLINIÉ F., JOLIVET A., Efficaces à tout âge ? Evolution démographique et activités de travail, Centre d'études de l'emploi, 2000, dossier 16. 
ANNEXE 1 : Caractéristiques de la population étudiée $(n=826)$

\section{Variable}

Groupe d'âge

$\leq 34$ ans.

$35-44$ ans.

$45-54$ ans.

$\geq 55$ ans.

\section{Genre}

Hommes

Femmes

\section{Statut social}

Ouvriers

Employés

Cadres inférieurs

Cadres supérieurs

Niveau d'étude

Primaire

Technique

Secondaire

Supérieur

\section{Secteurs d'activité}

Industrie chimique

Fabrication de machines

Industrie métallurgique- voitures

Construction

Commerce en gros

Horeca

Secteur financier

Recherche et développement

Administration publique

Enseignement

Santé

Secteur social
187

221

239

179

443

383

235

240

193

158

108

228

70

418

$\%$

$\%$

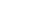

73

43

79

51

70

40

38

52

98

22,6

26,8

28,9

21,7

53,6

46,4

28,5

29,1

23,4

19,1

93

94

95

13,1

27,7

8,5

50,7

8,8

5,2

8,5

6,1

8,4

4,9

4,6

6,3

11,9

11,3

11,4

11,5 


\section{ANNEXE 2 : Présentation des stéréotypes sociaux liés à l'âge}

\begin{tabular}{|c|}
\hline Les travailleurs plus âgés .... \\
\hline s'investissent moins dans leur travail \\
\hline . sont plus heureux lorsqu'ils réduisent leur activité professionnelle \\
\hline . respectent mieux les règlements \\
\hline . s'adaptent moins facilement au changement \\
\hline Ce n'est plus la peine d'investir dans la formation des travailleurs plus âgés \\
\hline doivent coacher les plus jeunes \\
\hline . sont plus compétents du fait de leur expérience professionnelle \\
\hline . sont plus méticuleux \\
\hline . sont socialement isolés dans leur vie quotidienne \\
\hline . désirent quitter la vie professionnelle avant 65 ans \\
\hline . devraient laisser leur emploi aux plus jeunes \\
\hline . sont plus souvent absents \\
\hline . sont plus fidèles à l'entreprise \\
\hline .Les 5 sens (vue, audition, goût, toucher et odorat) s'affaiblissent avec l'âge \\
\hline .La capacité physique diminue avec l'âge \\
\hline . se fatiguent plus vite au travail \\
\hline . ont moins d'accidents de travail \\
\hline . sont moins efficaces, moins productifs \\
\hline . supportent moins bien les rythmes de travail élevés \\
\hline . savent plus facilement prendre des décisions seuls \\
\hline . nécessitent moins d'être encadrés dans leur travail \\
\hline . sont plus souvent déprimés \\
\hline .réagissent plus lentement face à l’imprévu \\
\hline . ont plus de problèmes de mémoire \\
\hline . sont plus créatifs \\
\hline . ont plus de problèmes de concentration \\
\hline . sont plus souvent de mauvaise humeur \\
\hline - préfèrent travailler seuls \\
\hline . mettent plus de temps pour apprendre quelque chose de nouveau \\
\hline . éprouvent plus de difficultés à utiliser les nouvelles technologies \\
\hline . sont plus sensibles au stress \\
\hline
\end{tabular}


Résumé : Tant la Belgique que la France se caractérisent par un faible taux d'emploi des seniors. Celui-ci peut être analysé en fonction de nombreux paramètres : politiques publiques de retrait anticipé, aspirations individuelles, dégradation des conditions de travail... Au niveau organisationnel, la présence de stéréotypes sociaux à l'égard du senior constitue également un facteur d'exclusion prématurée du marché de l'emploi. Cet article s'appuie sur des données récoltées auprès de 812 travailleurs, répartis dans douze secteurs d'activité différents. Les résultats de cette enquête montrent combien les stéréotypes négatifs à l'encontre des travailleurs âgés hantent le monde du travail. Ils sont portés par une jeune génération, plus formée, plus qualifiée et un secteur des services plus virulent. Mots-clés : Stéréotypes, discrimination, travailleur âgé, génération.

\begin{abstract}
Belgium and France are characterized by a low employment rate of older worker. This can be explained by different factors: public policies, individual aspirations, deteriorating working conditions... At the organizational level, social stereotypes are also a factor for exclusion from the labour market. This article is based on data collected from 812 workers, within 12 different activity sectors in Belgium. The results of this survey show that there are negative stereotypes towards the older workers and are worn by the youngest generation, more educated, and by the tertiary sector.
\end{abstract}

Keywords : stereotypes, discrimination, older worker, generation. 